\title{
STM observation of a single diarylethene flickering
}

\author{
N. Battaglini ${ }^{a}$ H. Klein ${ }^{a, *}$ C. Hortholary ${ }^{b}$ C. Coudret ${ }^{b}$ \\ F. Maurel ${ }^{\mathrm{c}} \mathrm{Ph}$. Dumas ${ }^{\mathrm{a}}$ \\ ${ }^{a}$ CRMC-N, Aix-Marseille Université Faculté des Sciences de Luminy, 13288 \\ Marseille cedex 9 \\ ${ }^{\mathrm{b}}$ CEMES, 29 rue Jeanne Marvig, BP 94347, 31055 Toulouse cedex 4 \\ ${ }^{\mathrm{c}}$ ITODYS, 1 rue Guy de la Brosse, 75005 Paris
}

\begin{abstract}
We report the STM observation of single diarylethene derivatives (DD) embedded into alkylthiol Self Assembled Monolayers (SAM) on $\mathrm{Au}(111)$. Telegraph noise in the data shows that the molecular conductance oscillates between two states. Comparing our results to the ones obtained by other teams observing conductance flickering with systems in the same geometry, we relate the two observed states to the two isomeric configurations of the molecule under study.
\end{abstract}

Key words: Scanning tunneling microscopy, Self assembly, Electronic transport in nanoscale materials and structures

PACS: 68.37.Ef, 81.16.Dn, 73.63.-b

\section{Introduction}

In the last thirty years, since Aviram's and Ratner's kick off proposal [1], a lot of theoretical and experimental work has been devoted to electron transport through molecules. Among the experimental techniques, scanning tunneling microscopy (STM) and derived techniques are of particular interest while, potentially, they allow access to electron transport studies of single molecules onto surfaces. However, measurements are not straightforward. One critical point is to immobilize the molecule onto the surface. This is often performed

\footnotetext{
* corresponding author

Email address: klein@crmcn.univ-mrs.fr (H. Klein ).
} 


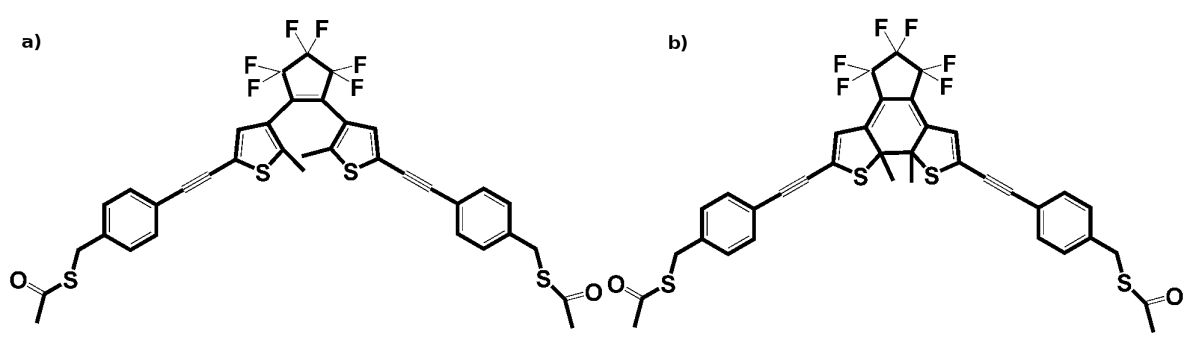

Figure 1. schematic representation of the two isomers of the diarylethene derivative under study. a) Open form. b) Closed form. Both are stable at room temperature. Both ends are functionalized by thioacetyl groups for anchorage on the $\mathrm{Au}(111)$ surface.

by cooling the sample down to cryogenic temperatures [2]. While surface diffusion is then hindered, the observer can peer at a particular molecule for long periods of time. Mechanically controlled break junctions (MCBJ) with molecules trapped at the junction are another way to measure electron transport through nanometer-sized single organic molecules $[3,4,5]$. Recently, in an insulating solvent at room temperature, STM has been operated as a MCBJ $[6,7,8]$. Although such STM junctions do not last for long, they last long enough to observe conduction through single molecular conduction channels. This allows an accurate measurement of the conductance of a single molecule by a statistical approach. As far as the time scale is concerned, our approach lies somewhere in between the two above mentioned techniques, allowing continuous observation of a single molecule for few tens of seconds.

Trapping the molecule in a 2D crystalline SAM hinders the molecule from diffusing on the surface, even at room temperature [9]. Continuous STM observations of a single molecule are then limited by the lateral thermal drift of the microscope ( $10 \mathrm{pm} / \mathrm{s})$.

The molecules we have been interested in for this study are diarylethene derivatives (DD) compounds [10] functionalized at both ends with thioacetyl groups. DD present two conformational isomers (see fig. 1). The diarylethene moiety is known for its photochromic ability [10,11]. Upon UV irradiation, the molecules photoisomerize in solution from a colourless open form (figure 1a) to a blue closed form (figure 1b). The reverse isomerization (from the closed to the open form) occurs upon visible irradiation. It is noteworthy that, in solution,DD are bistable molecules. Indeed, thermal interconversion between the two isomers is negligible at room temperature. DD are inserted in an octanethiol (C8) SAM deposited on $\mathrm{Au}(111)$ substrates. Beyond the advantage of preventing surface diffusion of DD, employing a SAM is expected to drive the orientation of the DD molecules embedded by the closely packed C8 chains which backbones are oriented $30^{\circ}$ away from the surface normal [12].

The STM observations of single DD presented hereafter demonstrate conductance flickering. The origin of this conductance flickering phenomenon for these bistable organic molecules is discussed. 


\section{Materials and methods}

Substrates are prepared under ultra high vacuum by evaporating gold onto freshly cleaved mica sheets maintained at $680 \mathrm{~K}$ (a more detailed description of the substrate preparation can be found elsewhere [9]). The resulting $\mathrm{Au}(111)$ surfaces are then immersed overnight in a millimolar dichloromethane solution containing both C8 and open DD isomer. The self-assembly mechanism (see e.g. [13]) occurring with this kind of systems leads to the formation of molecular 2D crystalline C8 areas. The samples are then rinsed with dichloromethane and dried under a nitrogen flow prior to STM analysis.

The samples are investigated in the dark, under ambient conditions with a customized low current STM developed on the basis of a commercial instrument. The STM tip is cut with scissors from a $0.25 \mathrm{~mm}$ Pt:Ir (80:20) wire. To minimize tip/molecule interactions [14], the junction impedance is typically settled to 300 G $\Omega$ (typical conditions : bias 1Volt, current 3pA).

Similarly to what is observed for alkylthiol SAMs on $\mathrm{Au}(111)$ surfaces [15], STM images of the samples immersed in the mixed DD:C8 solution show large crystalline $2 \mathrm{D}$ phases separated by disordered areas. (fig. 2 a). In addition to what is observed for alkylthiol SAMs on $\mathrm{Au}(111)$ surfaces, ordered domains of the mixed SAMs exhibit isolated bright features (fig. 2 b).

These bright features are unambiguously different from the defects commonly observed during STM imaging on alkylthiols SAMs. [15,16,17]. We assign these bright features to individual DD molecules.

In order to obtain images that routinely exhibit individual DD such as fig. 2 a, the DD:C8 ratio in solution has to be set to 20:1. The observed relatively low surface density of DD in well ordered areas is assigned to the difference in absorption kinetics and to demixion between the DD molecules and the C8 molecules [18].

In the following, we will focus on the individual DD molecules embedded in the 2D crystalline network of $\mathrm{C} 8$ and we will disregard the bunches of DD molecules grafted in the disordered areas (see caption of fig. 2). It is noteworthy that the bright features of fig. $2 \mathrm{~b}$ are not so homogenous and that, sometimes, one of them flickers from image to image albeit there is no evidence of tip change. In the next section, we discuss that point.

\section{Time dependent STM Measurements}

The main observation reported here is the reversible time-dependent STM contrast changes of these individual DD molecules. As stated in the previous paragraph, the shape of the individual molecules is not the same for all DD. 


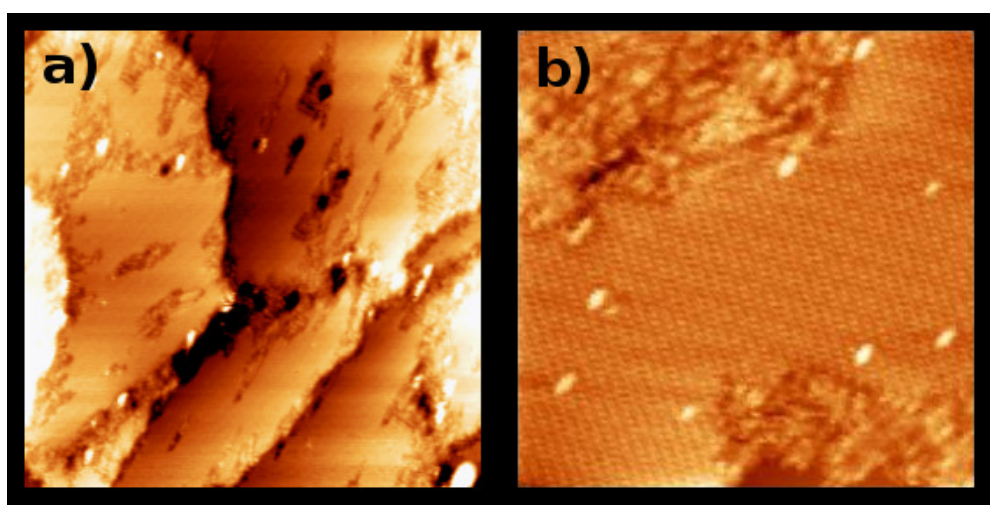

Figure 2. a) Low resolution STM image (width $=128 \mathrm{~nm}, \mathrm{~V}_{\text {bias }}=0.6 \mathrm{~V}, \mathrm{I}_{\text {tunnel }}=3 \mathrm{pA}$ ) illustrating the ordered and disordered areas of a mixed C8/DD SAM on $\mathrm{Au}(111)$. Smooth areas : ordered C8 SAM ; bright spots : DD bunches in disordered areas. b) High resolution STM image on the same sample (Width $=25.6 \mathrm{~nm}, \mathrm{~V}_{\text {bias }}=0.6 \mathrm{~V}$, $\mathrm{I}_{\text {tunnel }}=3 \mathrm{pA}$ ) showing individual DD inserted into a well ordered C8 SAM.

Moreover, STM contrast changes while scanning, without noticeable changes in the surrounding area of the molecule.

These changes occur with a time constant of the order of one second (data not shown). On one hand, this is too fast to be evidenced by successive STM images because the typical time needed for recording one image in our experimental conditions is 30s. On the other hand, because of the vertical drift, this is too rare to allow spectroscopic measurement in an open-loop mode. In order to gather experimental data of these changes of one individual molecule, we developed a specific technique. Within a STM image such as fig. 2, a single molecule is selected. To register a time dependent (denoted by $t$ ) cross section, we operate as follows. The fast scan axis ( $\operatorname{say} x$ ) is aligned parallel to the lateral drift direction to minimize its influence. The slow scan axis $(y)$ is disabled. A constant current $z(x, t) 2 \mathrm{D}$ image is then recorded (fig. 3), corresponding to a few tens of seconds of successive cross sections (typically $0.1 \mathrm{~s}$ each) of the isolated molecule.

Time profiles as well as histograms of the apparent height of a single DD in constant current mode are extracted from images such as the one shown in fig. 3 and shown in fig. 4 . The low conductivity of the SAM and the instrumental noise restrict the effective range of allowed bias and regulation current to narrow windows. Fig. 4 shows experiments performed at two different biases, 300 and $600 \mathrm{mV}$. To ensure that contrast changes are not due to experimental artifacts such as tip changes, we have also plotted a similar $z(t)$ profile recorded simultaneously on top of a nearby C8 molecule of the surrounding SAM. From these profiles, two observations can be made :

- the contrast changes are related to a "flickering" of the molecule between two defined states separated by an apparent height $\Delta z=0.1 \mathrm{~nm}$ without 


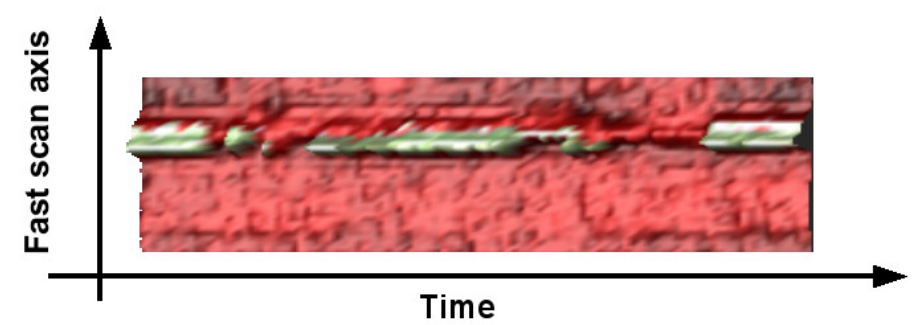

Figure 3. Typical time dependent STM profile $z(x, t)$ showing the reversible tunnel contrast changes of a single DD imbedded in a C8 SAM (horizontally : time scale is $16 \mathrm{~s}$; vertically, $x$ length scale is $4 \mathrm{~nm}$; $z$ length (colour scale) is $0.5 \mathrm{~nm}$; tunneling conditions are $\left.\mathrm{I}_{\text {reg }}=3 \mathrm{pA}, \mathrm{V}_{\text {bias }}=0.6 \mathrm{~V}\right)$.

To register such data, the fast scan axis $(x)$ is aligned parallel to the lateral drift direction ; the slow scan axis $(y)$ is disabled (see text).

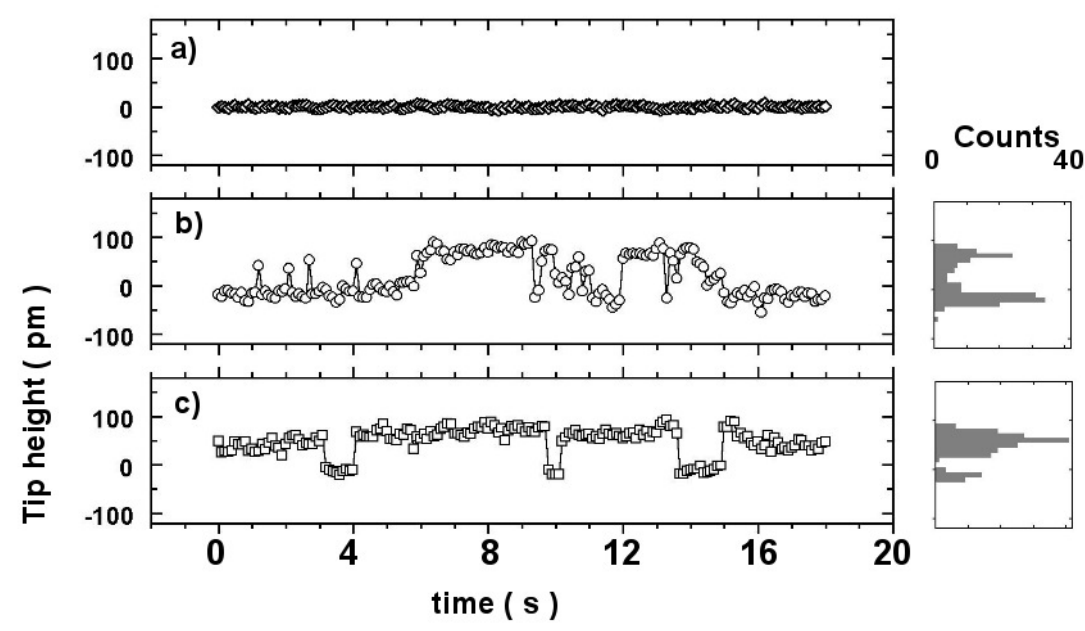

Figure 4. Time dependent and histograms of STM tip height over a single DD.Data are cross sections extracted from $z(x, t)$ measurements (such as shown in fig.3). a) cross section above a neighbooring $\mathrm{C} 8$ to show the stability of the tip. b) cross section above a single DD for a $300 \mathrm{mV}$ bias. c) cross section above a single DD for a 600 $\mathrm{mV}$ bias.

contrast changes of the surrounding SAM

- in the (narrow allowed) range $300-600 \mathrm{mV}$, and within the experimental noise, the applied bias seems to have an influence upon the relative times spent by the molecule in the "ON" and "OFF" states (as better shown by the histograms) but not on the value of $\Delta z$ 


\section{Discussion}

Although the experimental parameters (bias, current, temperature,...) could not be varied as widely as ideally expected, and although observing sequentially individual molecules with an ambient STM operated in the pA regime obviously limits the statistics, flickering of the DD molecules was demonstrated and deserves to be discussed in the light of the existing literature.

Time dependent conductance fluctuations have already been reported and interpreted in different ways. In the following, we discuss three important possible origins of conductance flickering in nano-objects. First we discuss the internal conformation change mechanism which we believe to be the more appropriate to describe our experiments. Then we discuss the possible contact quality change and electrostatic disturbance origins which are also likely to occur.

While studying a terphenyldithiol derivative molecule inserted inside an alkylthiol SAM, Weiss and co-authors [19] observed conductance flickering similar to our observation, though the time constant is typically one hour. The authors argue for a mechanism based upon internal conformation changes of the molecule. Indeed, qualitatively, when the aromatic cycles of one of their molecules are coplanar, the overlap of the orbitals is much more efficient resulting in a higher conductivity. More quantitatively, it has been shown [20] that the overlap between $\pi$ orbitals rapidly decays with an increasing dihedral angle between the aromatic moieties. These conformational changes of the polyaromatic molecules studied by the Weiss group lead to the measured STM height contrast related to "ON" and "OFF" states. Actually, our DD molecule is intrinsically bistable as it presents two stable isomers with different optical and electronic properties. Moreover, it is known that this bistability is neither hindered in the solid phase [10,21], nor when DD are grafted onto a gold surface [22]. The switch between the two isomeric forms is conveniently performed by a reversible photochemical process (photoisomerisation). In this light assisted mechanism, a $3 \mathrm{eV}$ photon is required to promote the open isomer from the ground state to an excited electronic state. From this excited state, the system can relax into the ground state of one of the two isomers (open or closed).

The energy involved in our tunneling process operating in the dark is much lower than $3 \mathrm{eV}$. Another mechanism must be at the origin of the flickering behaviour of the DD.

The potential energy barrier between the two isomeric forms is much lower than the energy of the photon needed for the photoisomerisation. We have estimated this energy barrier height by an ab initio calculation [23]. The open 
and closed forms of diarylethene model as well as the corresponding transition states for thermal ring opening/closing reactions were fully optimized without symmetry restrictions (convergence criteria $=10^{-4}$ ) with the Hartree-Fock method and using the 6-31G(d) basis set [24,25] ( $\mathrm{HF} / 6-31 \mathrm{G}(\mathrm{d})$ calculations). The Gaussian 94 program [26] was used for all calculations. Vibrational frequencies were calculated at the same level of theory to characterize the optimized structures as a minimum or a first-order saddle point on the potential energy surface (the Hessian matrix revealed a single negative eigenvalue for each transition state).

The energy barrier for ring-closure (cyclization) was found $0.6 \mathrm{eV}$ while the activation energy for cycloreversion is calculated near $0.3 \mathrm{eV}$. The results are summarized in fig. 5. These values are fully consistent with the fact that room temperature isomerization by thermal activation is highly improbable. While neither photons (experiments were carried out in the dark) neither thermal activation can explain isomerisation, another source of energy must be taken into account. We suggest that the energy required to overcome the potential barrier is brought by the tunneling electrons which carry, in our STM conditions $\left(I_{\text {reg }}=3 p A, V_{\text {bias }}\right.$ ranging from $0.3 \mathrm{~V}$ to $\left.1 \mathrm{~V}\right)$, each, an energy of the same order of magnitude.

Another explanation for conductance flickering in molecular systems has been proposed by the Lindsay's group [27] which reported reversible tunnel contrast modifications for alkyldithiols embedded into alkylthiol SAMs. It is proposed there that geometry changes of the thiol-surface bond affect the quality of the contact and are responsible of the flickering effect. This possibility is not likely to occur in our system while our studies are deliberately restricted to molecules inserted in well ordered areas, far from grain boundaries of the 2D SAM. The number of degrees of freedom is then reduced. By the way, in our previous studies [14,28], we have never observed such a phenomenon for molecules embedded in an ordered SAM matrix (namely mercapto-undecanol in an octanethiol matrix [28]).

The last mechanism to consider is known as "offset charge" in the field of Coulomb blockade devices. Systems of nanometric size having a very small capacitance are consequently highly sensitive to any electrostatic disturbance. Shall the electrostatic environment of our molecule be modified (e.g. by diffusing species), the energetic positions of the molecular levels, and thus the transport properties, will be. This uncontrollable phenomenon is general and affects either single electron transistors or Coulomb blockade based systems $[29,30]$.

In the "offset charge" mechanism, the fluctuation process, driven by fluctuations of the environment, is expected to be stochastic and uncontrollable. Such a mechanism would therefore be only poorly compatible with the observed bistable fluctuations evidenced in fig. 4 . 


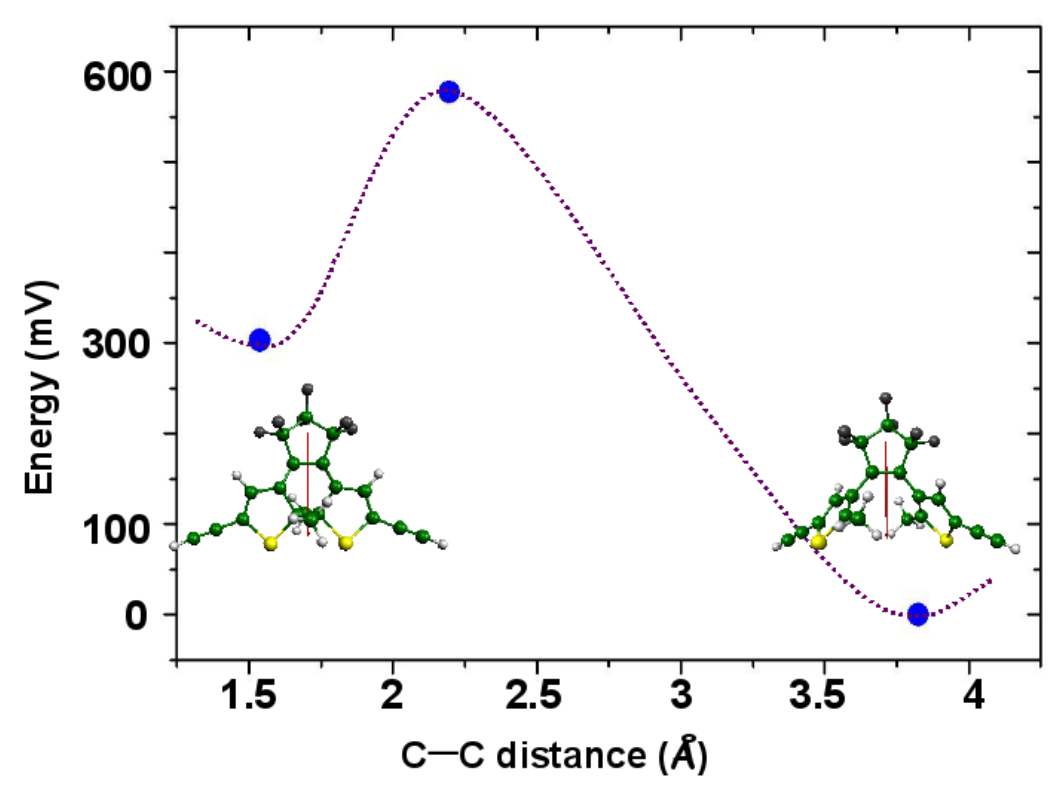

Figure 5. Energy of the DD molecule vs. the reaction coordinate (C-C distance) extracted from ab initio calculations.

Configurations are fully optimized without symmetry restrictions (see text for details).

The energy barrier for ring-closure (cyclization) was found $0.6 \mathrm{eV}$ while the activation energy for cycloreversion is calculated near $0.3 \mathrm{eV}$.

As mentionned in the introduction, the newly developped repetitive break junction method is a powerfull tool for measuring single molecule resistances. Recently, the results of such measurements on the two isomers of the DD molecule have been published by the Lindsay group [8]. They demonstrate that the conductance of the closed isomer is higher than the conductance of the open isomer. With the time constant of such experiments (few tens of $\mathrm{ms}$ ) and with the bias they use (always below $0.2 \mathrm{~V}$ ), they do not observe switching of a single molecule. While it removes the intrinsic limitations of low-current STM, this technique is however the most promising to observe controlled switching of a single molecule either by photon excitation either or by voltage pulses to higher bias values.

\section{5 conclusions}

We have shown that individual diarylethene derivatives embedded in an octanethiol SAM matrix flicker, exhibiting reversible bistable conductance fluctuations (labeled "ON" and "OFF") during STM experiments. 
Neither photons (experiments were carried out in the dark) neither thermal activation can explain isomerisation. Another source of energy must be taken into account. We make the proposal that the required energy is brought by the hot (few hundreds of meV) tunneling electrons. Our results suggest that the STM tip / sample bias influences the process.

This rises exciting possibilities for controlling the switching process of a single molecule electrically.

\section{References}

[1] Aviram A. and Ratner M. A., Chem. Phys. Lett., 29, 1974, 277.

[2] Qiu X. H., Nazin G. V. and Ho W., Phys. Rev. Lett., 93, 2004, 196806

[3] Smith R. H. M., Noat Y., Lang N.D., van Hemert M.C. and van Ruitenbeek J.M., Nature, 419, 2002, 906.

[4] Kergueris C., Bourgoin J. -P., Palacin S., Esteve D., Urbina C., Magoga M. and Joachim C., Phys. Rev. B, 59, 1999, 12505.

[5] Dulic D., van der Molen S.J., Kudernac T., Jonkman H.T., de Jong J.J.D., Bowden T.N., van Esch J., Feringa B.L. and van Wees B.J. Phys. Rev. Lett., 91, 2003, 207402

[6] Xu B. and Tao N. J., Science., 301, 2003, 1222.

[7] Haiss W., Nichols R.J., van Zalinge H., Higgins S.J., Bethell D. and Schiffrin D.J., Phys. Chem. Chem. Phys., 6, 2004, 4330.

[8] He J., Chen F., Liddell P.A., Andréasson J., Straight S. D., Gust D., Moore T. A., Moore A. L., Li J., Sankey O. F. and Lindsay S. M., Nanotechnology, 16, 2005, 695 .

[9] Klein H., Blanc W., Pierrisnard R., Fauquet C. and Dumas Ph., Eur. Phys. J. B, 14, 2000, 371 .

[10] Irie M., Chem. Rev., 100, 2000, 1685.

[11] Matsuda K. and Irie M., Polyhedron, 20, 2001, 1391.

[12] Ulman A., Chem. Rev., 96, 1996, 1533.

[13] Poirier G. E. and Pylant E. D., Science, 272, 1996, 1145.

[14] Klein H., Battaglini N., Bellini B. and Dumas Ph., Materials Science and Engineering C, 19, 2002, 279.

[15] Poirier G. E., Chem. Rev., 97, 1997, 1117. 
[16] Schonenberger C., Jorritsma J., Sondag-Huethorst J. A. M. and Fokkink L. G. J., J. Phys. Chem., 99, 1995, 3259.

[17] Bumm L. A., Arnold J. J., Charles L. F., Dunbar T. D., Allara D. L. and Weiss P. S., J. Am. Chem. Soc., 121, 1999, 8017.

[18] Ishida T., Mitzutani W., Choi N., Akiba U., Fujihira M. and Tokumoto H., J. Phys. Chem. B, 104, 2000, 11680.

[19] Donhauser Z. J., Mantooth B. A., Kelly K. F., Bumm L. A., Monnell L. D., Stapleton J. J., Price Jr. D. W., Rawlett A. M., Allara D. L., Tour J. M. and Weiss P. S., Science, 292, 2001, 2303.

[20] Woitellier S., Launay J. P. and Joachim C., Chem. Phys., 131, 1989, 481.

[21] Chen Q., Hiraga T., Men L., Tominaga J. and Atoda N., journal of Luminescence, 87-89, 2000, 776.

[22] Matsuda K., Ikeda M. and Irie M., Chem. Lett., 33, 2004, 456.

[23] Private communication. The details of the calculations performed will be the subject of a separate publication.

[24] Hariharan P. C. and Pople J. A., Chem. Phys. Lett., 66, 1972, 217.

[25] Hehre W. J., Radom L., Schleyer P. v. R. and Pople J. A., Ab Initio Molecular Orbital Theory, Wiley, New York, 1986.

[26] Frisch M. J., Trucks G. W., Schlegel H. B. et al., Gaussian 94, Revision E.1, Gaussian, Inc.: Pittsburgh, PA, 1995.

[27] Ramachandran G. K., Hopson T. J., Rawlett A. M., Nagahara L. A., Primak A. and Lindsay S. M., Science, 300, 2003, 1413.

[28] Battaglini N., Klein H., Dumas Ph., Moustrou C. and Samat A., Appl. Surf. Sci., 212-213, 2003, 481.

[29] Grupp D. E., Zhang T., Dolan G. J. and Wingreen N. S., Phys. Rev. Lett., 87, 2001, 186805.

[30] Gautier J., Microelectronic Engineering, 39, 1997, 263. 